\title{
Deep-space laser-ranging missions ASTROD and ASTROD I for astrodynamics and astrometry
}

\author{
W. T. Ni and the ASTROD I ESA \\ COSMIC VISION 2015-2025 TEAM \\ Center for Gravitation and Cosmology, Purple Mountain Observatory, \\ Chinese Academy of Sciences, Nanjing, 210008, China, \\ email: wtni@pmo.ac.cn
}

\begin{abstract}
Deep-space laser ranging will be ideal for testing relativistic gravity, and mapping the solar-system to an unprecedented accuracy. ASTROD (Astrodynamical Space Test of Relativity using Optical Devices) and ASTROD I are such missions. ASTROD I is a mission with a single spacecraft; it is the first step of ASTROD with 3 spacecraft. In this talk, after a brief review of ASTROD and ASTROD I, we concentrate on the precision of solar astrodynamics that can be achieved together with implications on astrometry and reference frame ties. The precise planetary ephemeris derived from these missions together with second post-Newtonian test of relativistic gravity will serve as a foundation for future precise astrometry observations. Relativistic frameworks are discussed from these considerations.
\end{abstract}

Keywords. astrometry, gravitation, relativity, reference systems, celestial mechanics

\section{Introduction}

Improvement of the accuracy of Satellite Laser Ranging (SLR) and Lunar Laser Ranging (LLR) has a great impact on the geodesy, reference frames and test of relativistic gravity. At present, the accuracy for 2-color (2-wavelength) satellite laser ranging reaches $1 \mathrm{~mm}$ and that for 2-color lunar laser ranging are progressing towards a few millimeters. The present SLR and LLR are passive ranging; the intensity received at the photodetector is inversely proportional to the fourth power of the distance; for LLR, the received number of photons is low. However, for active ranging in which the laser light is received at the other end of ranging, the intensity received is inversely proportional to the second power of distance. Even at interplanetary distances, the received photons would be abundant and deep-space laser ranging missions are feasible. The primary objective for the long-term ASTROD (Astrodynamical Space Test of Relativity using Optical Devices) concept is to maintain a minimum of 3 spacecrafts in orbit within our solar system using laser interferometric ranging to ultimately test relativity and to search for gravitational waves. This is divided into 3 distinct stages, each with increasing order of scientific benefits and engineering milestones. The first stage, ASTROD I, will comprise a single spacecraft communicating with ground stations using laser pulse ranging. Fig. 1 (left) gives a schematic of the proposed orbit design. The second phase, ASTROD II, also called ASTROD since it is a testbed of the general ASTROD mission concept, will consist of three spacecrafts ( 2 in solar orbits, one at the Sun-Earth Lagrangian point L1 [or L2]) communicating with 1-2 W CW lasers. Fig. 1 (right) depicts the orbit design and configuration of the spacecraft 700 days after launch. The third and final stage, ASTROD III or Super ASTROD, will then explore the possibility of larger orbits in an effort to detect lower frequency primordial gravitational waves. 

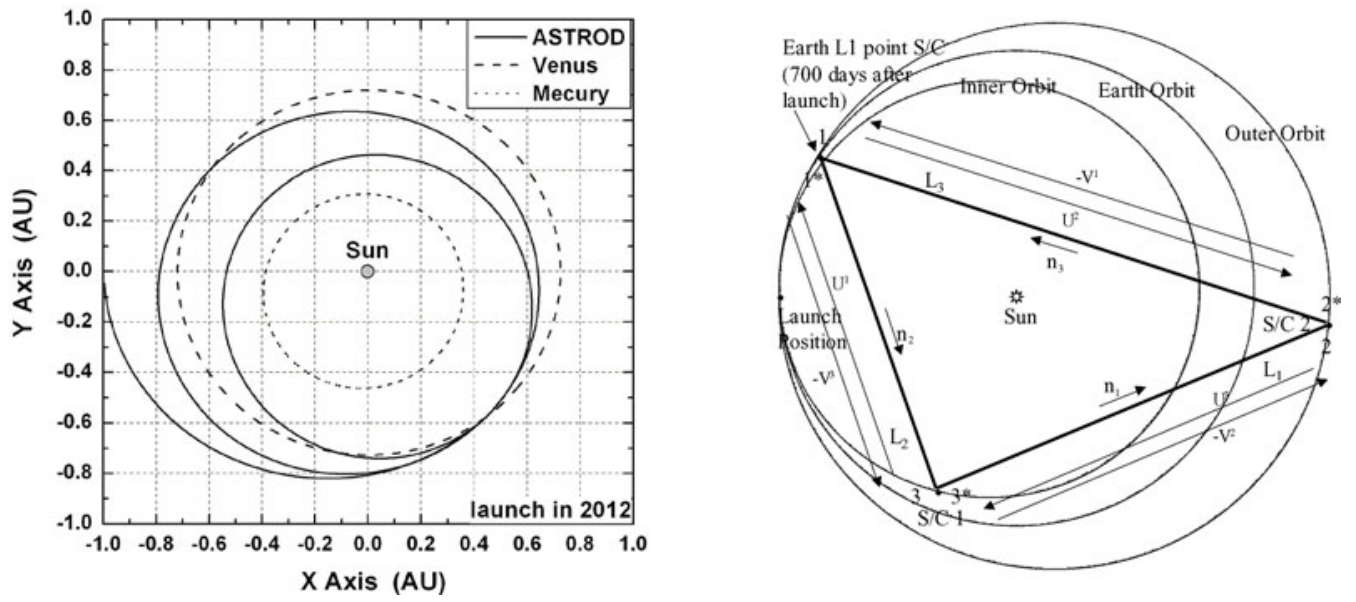

Figure 1. (left) A 2012 orbit in heliocentric ecliptic coordinate system for ASTROD I spacecraft; (right) A schematic ASTROD configuration (baseline ASTROD after 700 days from launch).

Table 1. Summary of the scientific objectives of the ASTROD I mission.

\begin{tabular}{|c|c|c|}
\hline Effect/Quantity & Present accuracy & | Projected accuracy \\
\hline PPN parameter $\beta$ & $2 \times 10^{-4}$ & $3 \times 10^{-8}$ \\
\hline PPN parameter $\gamma$ & $4.4 \times 10^{-5}$ & $3 \times 10^{-8}$ \\
\hline Lense Thirring Effect & 0.1 & 0.1 \\
\hline$(\mathrm{dG} / \mathrm{dt}) / \mathrm{G}$ & $10^{-12} \mathrm{yr}^{-1}$ & $3 \times 10^{-14} \mathrm{yr}^{-1}$ \\
\hline Anomalous Pioneer acceleration $A_{a}$ & $(8.74 \pm 1.33) \times 10^{-10} \mathrm{~m} / \mathrm{s}^{2}$ & $0.7 \times 10^{-16} \mathrm{~m} / \mathrm{s}^{2}$ \\
\hline Determination of solar quadrupole moment & $(1-3) \times 10^{-7}$ & $1 \times 10^{-9}$ \\
\hline Determination of planetary masses and orbit parameters & (depends on object) & 1-3 orders better \\
\hline Determination of asteroid masses and densities & (depends on object) & $2-3$ orders better \\
\hline
\end{tabular}

\section{ASTROD I}

The basic scheme of the ASTROD I space mission concept as proposed to ESA Cosmic Vision 2015-2025 is to use two-way laser pulse ranging between the ASTROD I spacecraft in solar orbit and deep space laser stations on Earth to improve the precision of solar-system dynamics, solar-system constants and ephemeris, to measure the relativistic gravity effects and test the fundamental laws of spacetime more precisely, and to improve the measurement of the time rate of change of the gravitational constant. A summary of ASTROD I goals is compiled in Table 1 (Appourchaux et al. (2007)).

To achieve these goals, the timing accuracy for ranging is required to be less than 3 ps and the drag-free acceleration noise at $100 \mu \mathrm{Hz}$ is required to be below $3 \times 10^{-14} \mathrm{~m}$ $\mathrm{s}^{-2} \mathrm{~Hz}^{-1 / 2}$. Figure 2 (left) shows the drag-free acceleration noise requirements for ASTROD I, the LTP, LISA and ASTROD for comparisons. To separate astrodynamic effect from relativistic-gravity test, the ASTROD I orbit is designed to reach the opposite side of the Sun in about 365 day and 680 day from launch. The apparent angles of the spacecraft during the two solar oppositions are shown in Fig. 2 (right) for the 2012 trajectory in Fig. 1. The maximum one-way Shapiro time delays near the two solar oppositions are 

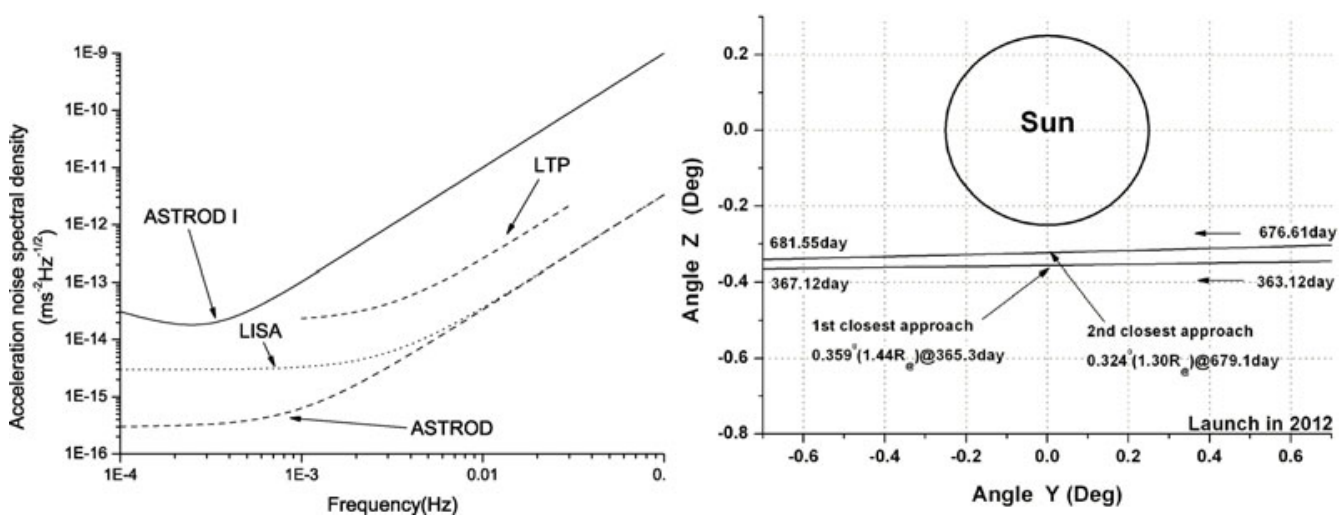

Figure 2. (left) A comparison of the target acceleration noise curves of ASTROD I, the LTP, LISA and ASTROD; (right) Apparent angles during the two solar opposition for the ASTROD I orbit in figure 1.

Table 2. Summary of the astrodynamical and astrometric objectives of the ASTROD I and ASTROD missions.

\begin{tabular}{|c|c|c|}
\hline Effect/Quantity & ASTROD I & ASTROD \\
\hline Timing accuracy & $3 \mathrm{ps}$ & 1 ps or better \\
\hline Timing precision & $3 \mathrm{ps}$ & $0.1 \mathrm{ps}$ \\
\hline Ranging accuracy & $0.9 \mathrm{~mm}$ & $0.3 \mathrm{~mm}$ or better \\
\hline Ranging precision & $0.9 \mathrm{~mm}$ & $3-10 \mu \mathrm{m}$ \\
\hline Acceleration noise at $100 \mu \mathrm{Hz}$ & $3 \times 10^{-14} \mathrm{~m} \mathrm{~s}^{-2} \mathrm{~Hz}^{-1 / 2} \mid 3$ & $10^{-16} \mathrm{~m} \mathrm{~s}^{-2} \mathrm{~Hz}^{-1 / 2}$ \\
\hline Derived angle accuracy & $<10 \mu$ as & $1 \mu$ as \\
\hline Dynamical frame accuracy & $<100 \mu$ as & $<10 \mu$ as \\
\hline
\end{tabular}

$0.1172 \mathrm{~ms}$ (in $365.3 \mathrm{day}$ ) and $0.1196 \mathrm{~ms}$ (in 679.1 days), respectively. The Shapiro time delay can be measured precisely to give a good test of relativistic gravity.

Table 2 summarizes the astrodynamical and astrometric objectives of the ASTROD I and ASTROD missions.

The angle accuracy depends on (i) derived angle accuracy from ranging of ASTROD I spacecraft and (ii) laser pointing measurement noise. With a ranging accuracy of $0.9 \mathrm{~mm}$ and acceleration noise as quoted, the derived azimuthal range accuracy would be much better than $1 \mathrm{~m}$. This corresponds to $1.3 \mu$ as in angle at a distance of 1 AU. For laser pointing measurement accuracy, we assume that it is below $10 \mu$ as. This assumption gives the derived angle accuracy below $10 \mu$ as (Table 2). For the dynamical frame accuracy, we conservatively down grade it by a factor of 10 to below $100 \mu$ as.

Just like LLR, ASTROD I (and ASTROD) will contribute to the determination of celestial reference frame, terrestrial frame and earth rotation. A dynamical realization of the International Celestial Reference System (ICRS) by the lunar orbit has been obtained from LLR to an uncertainty about 1 mas. A similar determination from ASTROD I orbit would lead to an uncertainty below $100 \mu$ as. The simultaneous data-fitting solution for the coordinates and velocities for the laser ranging stations will contribute to the realization of international terrestrial reference frame. Ranging to ASTROD I would contribute to the determination of nutation parameters due to the dynamical stability of ASTROD I orbit. 


\section{ASTROD}

ASTROD (ASTROD II) mission concept is to have two spacecraft in separate solar orbits carrying a payload of a proof mass, two telescopes, two $1-2 \mathrm{~W}$ lasers, a clock and a drag-free system, together with a similar spacecraft near Earth at one of the Lagrange points L1/L2 (Bec-Borsenberger et al. 2000; Ni et al. 2004). The three spacecrafts range coherently with each other using lasers to map the solar-system gravity, to test relativistic gravity, to observe solar g-mode oscillations, and to detect gravitational waves. Distances between spacecraft depend critically on the solar-system gravity (including gravity induced by solar oscillations), underlying gravitational theory and incoming gravitational waves. A precise measurement of these distances as a function of time will determine these causes. After 2.5 years, the inner spacecraft completes 3 rounds, the outer spacecraft 2 rounds, and the L1/L2 spacecraft (Earth) 2.5 rounds. At this stage two spacecraft will be on the other side of the Sun, as viewed from the Earth, for conducting the Shapiro time delay experiment efficiently. The spacecraft configuration after 700 days from launch is shown in Figure 1(right).

For a mission like ASTROD II within the next 10 - 20 years, the timing accuracy better than 1 ps $(300 \mu \mathrm{m}$ in terms of range) can be anticipated. In coherent interferometric ranging, timing events need to be generated by modulation/encoding technique or by superposing timing pulses on the $\mathrm{CW}$ laser light. The interference fringes serve as consecutive time marks. With timing events aggregated to a normal point using an orbit model, the precision can reach $30 \mu \mathrm{m}$ in range. The effective range precision for parameter determination could be better, reaching $3-10 \mu \mathrm{m}$ by using orbit models. Since ASTROD range is typically of the order of $1-2 \mathrm{AU}\left((1.5-3) \times 10^{11} \mathrm{~m}\right)$, a range precision of $3 \mu \mathrm{m}$ will give a fractional precision of distance determination of $10^{-17}$. Therefore, the desired clock accuracy/stability is $10^{-17}$ over $1000 \mathrm{~s}$ of travel time. Optical clocks with this accuracy/stability are under development. The space optical clock is under development for the Galileo project. This development would pave the road for ASTROD to use optical clocks. With these anticipations, we list the astrodynamical and astrometric objectives in Table 2 .

\section{Relativistic frameworks}

For the relativistic framework to test relativity and to do astrodynamics and astrometry, we use the scalar-tensor theory of gravity including intermediate range gravity which we used in obtaining the second post-Newtonian (2 PN) approximation (Xie et al. 2007). For the light deflection in the $2 \mathrm{PN}$ approximation in the solar field, please see Dong \& $\mathrm{Ni}$ (2007). To extend to multi-bodies with multipoles in the solar system, we will extend our calculation following the work of Kopeikin \& Vlasov (2004).

We thank National Natural Science Foundation of China (Grant Nos 10475114 and 10778710) and Foundation of Minor Planets of Purple Mountain Observatory for support.

\section{References}

Appourchaux, T., et al. 2007, ASTROD I: proposal for ESA Cosmic Vision 2015-2025

Bec-Borsenberger, A., et al. 2000, ASTROD-A Proposal for Two ESA Flexi-Mission F2/F3

Dong, P. \& Ni, W.-T. 2008, Light deflection in the second post-Newtonian approximation of scalar-tensor theory of gravity, this volume, p.401

Kopeikin, S. \& Vlasov, I. 2004, Phys. Rep., 400, 209

Ni, W.-T., Shiomi, S., \& Liao, A.-C. 2004, Class. Quantum Grav., 21, S641

Xie, Y., Ni, W-T., Dong, P., \& Huang, T-Y. 2007, Adv. Spac. Res., doi: 10.1016/j.asr.2007.09.022; arXiv:0704.2991 